\title{
HIV and tuberculosis in India
}

\author{
Soumya Swaminathan* and G Narendran \\ Deptartment of Clinical Research, Tuberculosis Research Centre, Mayor VR Ramanathan Road, Chetput, \\ Chennai 600 031, India \\ *Corresponding author (Fax 91-44-2836 2528 ; Email, doctorsoumya@yahoo.com)
}

\begin{abstract}
The global impact of the converging dual epidemics of tuberculosis (TB) and human immunodeficiency virus (HIV) is one of the major public health challenges of our time. The World Health Organization (WHO) reports 9.2 million new cases of TB in 2006 of whom 7.7\% were HIV-infected. Tuberculosis is the most common opportunistic infection in HIV-infected patients as well as the leading cause of death. Further, there has been an increase in rates of drug resistant tuberculosis, including multi-drug (MDRTB) and extensively drug resistant TB (XDRTB), which are difficult to treat and contribute to increased mortality. The diagnosis of TB is based on sputum smear microscopy, a 100-year old technique and chest radiography, which has problems of specificity. Extra-pulmonary, disseminated and sputum smear negative manifestations are more common in patients with advanced immunosuppression. Newer diagnostic tests are urgently required that are not only sensitive and specific but easy to use in remote and resource-poor settings. Treatment of HIV-TB co-infection is complex and associated with high pill burden, overlapping drug toxicities, risk of immune reconstitution inflammatory syndrome (IRIS) and challenges related to adherence. From a programmatic point of view, screening of all HIV-infected persons for tuberculosis and vice-versa will help identify co-infected patients who require treatment for both infections. This requires good coordination and communication between the TB and AIDS control programs, in India.
\end{abstract}

[Swaminathan S and Narendran G 2008 HIV and tuberculosis in India; J. Biosci. 33 527-537]

\section{Introduction}

The increasing rate of human immunodeficiency virus (HIV) infection in many countries has had an impact on tuberculosis (TB) epidemiology. While TB prevalence has remained stable, TB incidence continues to rise, especially in countries most severely affected by the HIV epidemic as well as those facing political turmoil, migration, poverty and unemployment and where intravenous drug abuse is rampant. $\mathrm{HIV}$ is the most important known risk factor that promotes progression to active TB in people with Mycobacterium tuberculosis infection (TB/HIV A Clinical Manual 2004). The lifetime risk of tuberculosis in immunocompetent persons is $5 \%$ to $10 \%$, but in HIV positive individuals, there is a $5 \%$ to $15 \%$ annual risk of developing active TB disease (Swaminathan et al 2000). WHO estimated 9.2 million new cases of TB globally in 2006 (139 per 100,000); of whom 7,09,000 (7.7\%) were HIV positive (World Health Organization 2008). India, China, Indonesia, South Africa and Nigeria rank first to fifth in terms of incident TB cases.

In India, there were 2.5 million people living with HIV and AIDS (PLWHA) at the end of 2007 while the incidence of TB was approximately 1.8 million cases per year (WHO Release 2007, RNTCP 2008). In a survey carried out among new tuberculosis patients by the Revised National TB control Program (RNTCP) in 2007, HIV sero-prevalence

Keywords. Clinical features; control program; HIV; India; tuberculosis

Abbreviations used: DMC, designated microscopy centre; HIV, human immunodeficiency virus; ICTC, integrated counseling and testing centre; IRIS, immune reconstitution inflammatrory syndrome; NRTIs, nucleoside reverse transcriptase inhibitors; NSAID, non-steroidal anti-inflammatory drug; PCP, Pneumocystis Jiroveci pneumonia; PGL, persistent generalized lymphadenopathy; PI, protease inhibitor; RNTCP, Revised National TB Control Program; TB, tuberculosis 
varied widely and ranged from $1 \%$ to $13.8 \%$ across the 15 districts (Central TB Division, unpublished observations). Currently, it is not clear what role the HIV epidemic has played in the TB situation in India. If HIV prevalence in the community continues to increase, however, it could affect the TB control program, by decreasing cure rates and increasing mortality and recurrent TB. Further, appropriate management of patients with TB/HIV requires not only treating the tuberculosis but referring them for CD4 count testing and anti-retroviral treatment, if indicated.

\section{Impact of HIV on TB manifestations}

HIV infection is associated not only with an increased incidence of TB, but with altered clinical manifestations especially in the advanced stages. As HIV progresses, there is cutaneous anergy as well as impaired tissue containment of mycobacteria leading to widespread dissemination of mycobacteria. Figure 1 shows the increasing risk of tuberculosis as CD4 declines. In patients with latent TB infection, the risk of developing active disease is higher among recently infected persons compared to those with chronic infection but is several hundred-fold higher among persons who acquire HIV (figure 2). Further, among persons with HIV infection, newly acquired TB infection can rapidly progress to active disease.

It is clear that HIV infection acquired after TB infection is a significant risk factor for development of active TB- mainly due to its effects on the immune system. HIV is associated with decreased chemotaxis, defective granuloma formation and maintenance, impaired antigen processing and presentation as well as generalized loss of $\mathrm{CD} 4+\mathrm{T}$ cells and selective clonal depletion of $M-T B$ specific CD4+ T lymphocytes (Lawn et al 2002). While TB can develop at any CD4 count, extrapulmonary and disseminated forms of the disease are more common as immunodeficiency increases.

Recently, Miranda et al (2007) demonstrated an $80 \%$ reduction in incident TB in HAART treated compared to ART-naïve HIV-infected persons in Brazil. It is likely that as access to ART improves world-wide, there will be a reduction in new TB cases, though rates would still be higher than in the general population.

\section{Pulmonary TB}

Pulmonary involvement occurs in about $75 \%$ of all HIVinfected patients with TB. (Deivanayagam et al 2001; Ahmad and Shameem 2005; TB/HIV: A Clinical Manual 2004). Unlike other opportunistic infections which have a selective range of CD4 in which the disease occurs, TB occurs throughout the course of HIV. The interaction between HIV and TB in persons coinfected with HIV and TB is bidirectional and synergistic. Clinical presentation however depends on the level of immnuosuppression. Typical manifestations are frequent in early HIV and atypical features begin to occur as the immunity declines with more frequent extra pulmonary manifestations ranging from 46 to $79 \%$ (Kumarasamy et al 2005; Sharma et al 2005). An HIV positive individual with any of the following symptoms should be suspected of having TB and investigated further: cough of more than 2 weeks duration, fever lasting more than

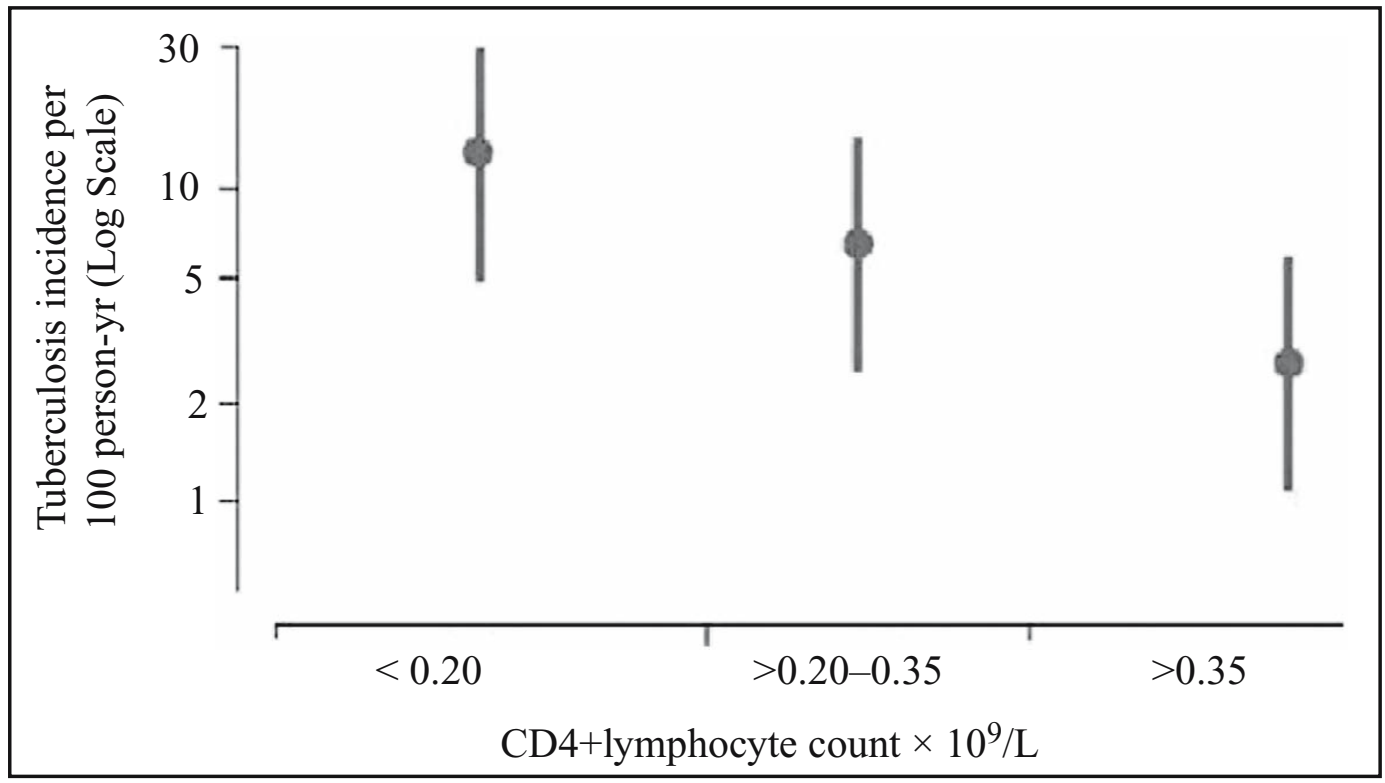

Figure 1. Risk of tuberculosis among HIV infected persons by CD4 + lymphocyte count (Antonucci et al 1995). 


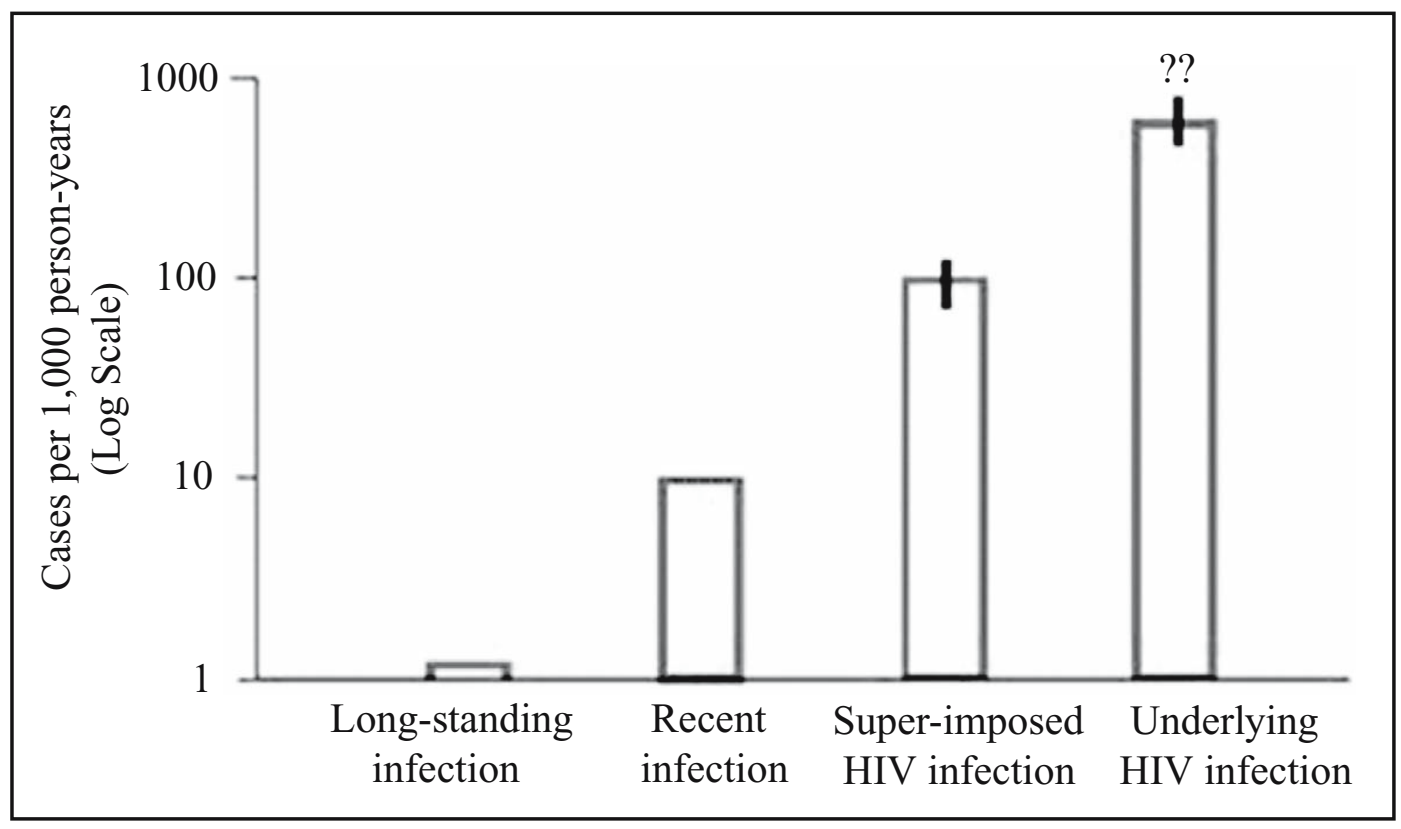

Figure 2. Risk of tuberculosis following tuberculous infection in individuals with chronic infection, recent infection and in those who acquire HIV infection (Rieder 1999).

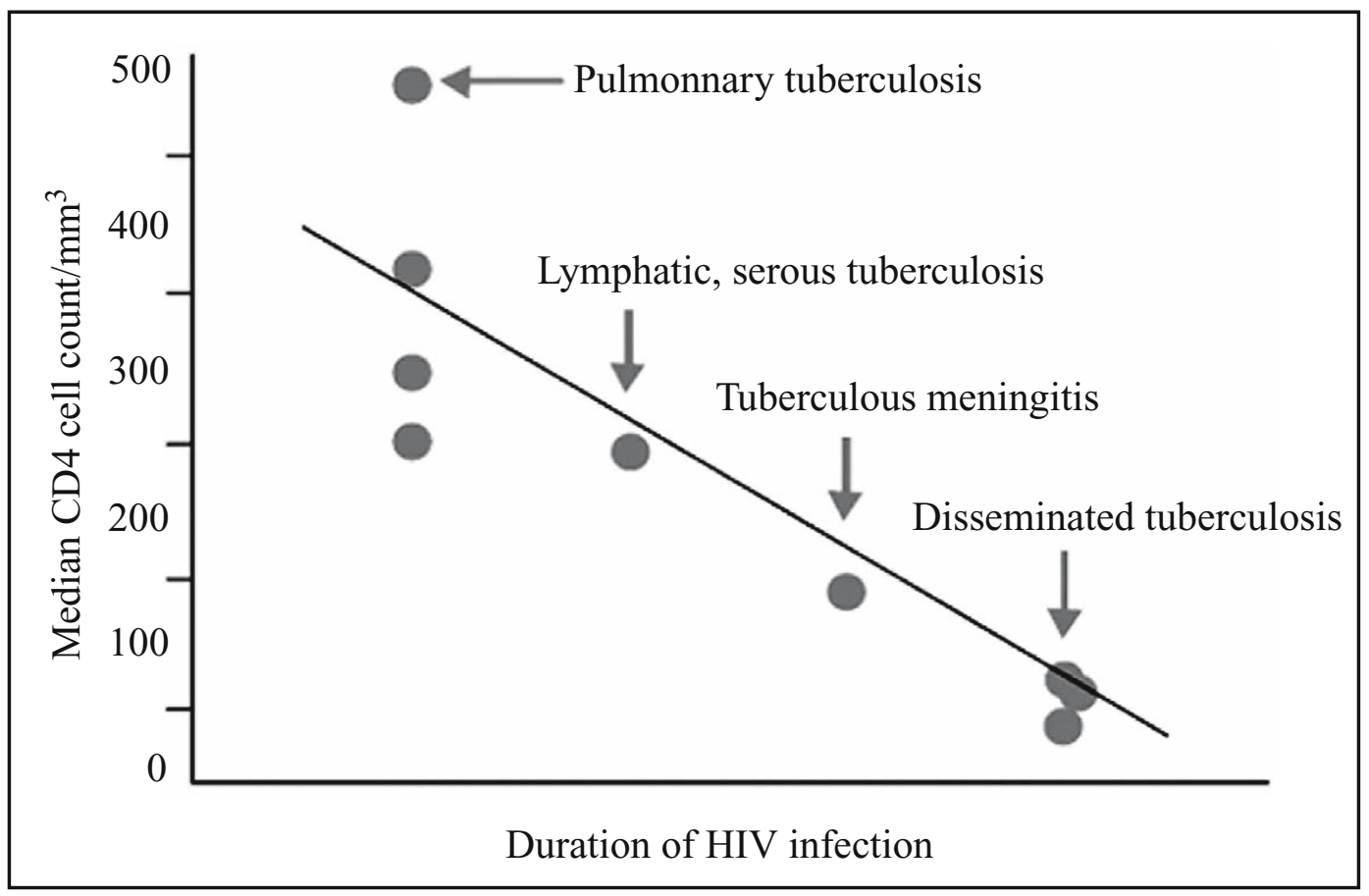

Figure 3. Clinical and immuno pathological course of HIV associated tuberculosis (De Cock et al 1992).

2-3 weeks, weight loss, fatigue, listlessness, unexplained dyspnoea, chest pain, or hemoptysis. Accelerated weight loss defined as greater than $10 \%$ weight loss in a month in HIV has earned the name "slimming disease". Differential diagnoses include bacterial or fungal pneumonias, Pneumocystis Jiroveci pneumonia (PCP), lymphoma and wasting syndrome. Cough with productive mucopurulent sputum is in favour of bacterial pneumonias including 
TB whereas nonproductive cough with dyspnoea out of proportion to signs is in favour of PCP (TB/HIV A Clinical Manual 2004).

\section{Extra pulmonary TB}

Extra pulmonary TB is the commonest cause of pyrexia of unknown origin (PUO) among HIV+ individuals in developing countries (Rupali et al 2003; Kejariwal et al 2001). The most frequent extra pulmonary form of TB is involvement of the lymph nodes with cervical region being the commonest (Arora and Kumar 1999). Persistent generalized lymphadenopathy (PGL) simulates TB of the nodes. In PGL, nodes are symmetrical, asymptomatic and non-tender whereas in TB, lymph nodes are usually larger, asymmetric, painless and matted and maybe associated with constitutional symptoms or mediastinal/hilar adenopathy in the chest x-ray. Intrathoracic lymphadenopathy is not a feature of primary HIV syndrome and should always the raise the suspicion of TB or lymphoma. Lymph node involvement in HIV is characterized by poor granuloma formation with abundant AFB picked up by Ziehl Neelsen stain (Havlir and Barnes 1999) Diagnosis can be easily made by FNAC (done as an out-patient procedure) or by lymph node biopsy. While Mycobacterium tuberculosis accounts for most mycobacterial infections in India, a small proportion are due to non-tuberculous mycobacteria like $M$. avium intracellulare, $M$. fortuitum etc. - molecular or high performance liquid chromatography (HPLC) assays are required to differentiate between species (Singh et al 2007). The other forms of extrapulmonary TB include pleural effusion, pericardial effusion, abdominal TB, TB meningitis and abdominal TB. Pleural effusion is usually unilateral and asymmetric, may be associated with parenchymal opacities.

Intra cranial space occupying lesions are common in HIV-infected individuals: the differential diagnosis includes cerebral toxoplasmosis, tuberculoma, neurocysticercosis, cryptococcoma and lymphoma. In India, cryptococcal meningitis is the commonest form of meningitis followed by TB meningitis. A finding that is strongly suggestive of disseminated TB is the detection of choroidal tubercles associated with miliary TB (Sahn and Neff 1974). Features of abdominal TB include hepatosplenomegaly with focal intra abdominal lymphadenopathy. Ascitis and omental thickening are less common in HIV (Fee et al 1995).

Mycobacteremia has been observed to be an important cause of PUO, particularly among patients with severe immunosuppression, but is difficult to diagnose as it requires facilities to perform blood culture using rapid culture techniques like BACTEC (Ramachandran et al 2002). In a study done in Africa, in patients with severe wasting at necropsy, nearly half had evidence of disseminated TB (Lucas et al 1994).

\section{Investigations for diagnosis of TB}

\subsection{Sputum examination}

Three sputum specimens should be collected for patients suspected to have TB, including at least one overnight collection. Smear should be stained by Ziehl-Neelsen (conventional microscopy) or Auramine rhodamine (fluorescent microscopy). The advantages of fluorescence over light microscopy for the detection of pulmonary TB have been confirmed in a systematic review of 45 studies comparing the 2 methods, which found that fluorescence microscopy yielded an average increase in sensitivity of $10 \%$ with no loss of specificity (Steingart et al 2006). In situations where all 3 smears are negative, the RNTCP recommends a course of broad-spectrum antibiotics (avoiding quinolones) and repeating sputum smear examinations and performing a chest $\mathrm{x}$-ray if the patient is still symptomatic for chest disease (Agarwal and Chauhan 2005). WHO recently proposed a modified algorithm for severely ill patients suggesting that anti-TB treatment can be started based on non-response to antibiotics and a chest x-ray, within 3-5 days (WHO smear negative algorithm 2006). However, this approach needs to be validated in a low HIV-prevalence country like India.

Culture (on Lowenstein-Jensen medium) is much more sensitive but takes 6-8 weeks and requires specialized laboratory facilities and trained staff. Automated liquid culture systems that detect bacterial $\mathrm{CO}_{2}$ production or $\mathrm{O}_{2}$ consumption with radiometric (BACTEC), fluorescent (MGIT), colorimetric (MB/BacT system) or pressure sensors (ESP culture system II) roughly halve the time to detection and are being evaluated for use in resource-poor settings (Williams-Bouyer et al 2000; Lee et al 2003; Gil-Setas et al 2004). Molecular assays have been developed for rapid detection of growth as well as species identification and drug susceptibility testing. The 3 most widely used assays, polymerase chain reaction (PCR) (Roche Diagnostics), transcription mediated amplification (Genprobe) and stranddisplacement amplification (Becton Dickinson) have shown excellent sensitivity, specificity and speed. Despite the clear advantages of these nucleic acid amplification tests over existing tests, especially for the rapid detection of smear negative disease, they have only limited use in TB endemic settings, primarily because of their cost and complexity.

\section{Chest radiography}

While radiography is a commonly performed investigation, it must be remembered that patients with HIV co-infection may not have typical radiographic features of pulmonary tuberculosis. While patients with higher CD4 cells $(>350$ cells $/ \mathrm{mm}^{3}$ ) have radiographic abnormalities similar to their 
HIV negative counterparts, patients with immunosuppression often have minimal or atypical findings (Long et al 1991). Diffuse pulmonary infiltrates/opacities are the dominant radiological presentation and cavitation is uncommon. Miliary pattern, mediastinal adenopathy and pleural effusion are more common and $\mathrm{x}$-rays may be normal in $5-10 \%$ of $\mathrm{HIV}+$ individuals (Swaminathan et al 2007). Differential diagnoses include PCP, non-tuberculous mycobacteria, nocardiosis, fungal infections and non-infective complications like lymphocytic interstitial pneumonitis. In the presence of a radiographic lesion suggestive of TB with negative sputum smears in an ambulatory patient, the best approach is to give a course of broad spectrum antibiotics (including co-trimoxazole) and repeat x-ray after 10-15 days. Lack of improvement or deterioration would indicate TB (TB/HIV A Clinical Manual 2004).

\section{Tuberculin skin (Mantoux) test}

Tuberculin skin reactivity is dependant on cytokine mediated cellular immunity. It has been used as a diagnostic test for tuberculosis infection: an induration of $5 \mathrm{~mm}$ or more, in response to $1 \mathrm{TU}$ of PPD-RT 23 tuberculin is usually taken as a positive response, in HIV-infected individuals. In TB high prevalence countries like India, tuberculin skin testing has no value in TB diagnosis, as a positive test indicates prior infection only and false negativity (due to anergy or other unknown factors) is common. In a recent analysis, we found that even with the cut off of $5 \mathrm{~mm}$ universally practiced for $\mathrm{TB}$ in $\mathrm{HIV}$, in India the positive predictive value of a TST for TB was $48.8 \%$ for patients with CD4 < 200 cells $/ \mathrm{mm}^{3}$ and only 18.8 for patients with CD4 $>200$ cells $/ \mathrm{mm}^{3}$ (Swaminathan et al 2008). Anergy testing using polyvalent antigens like candida, tetanus toxoid, or mumps has been given up now because it is highly unreliable. Overall, tuberculin testing is not useful in routine practice either for diagnosis of TB or latent TB infection. An initially negative PPD may become positive when IRS occurs and this has been suggested as a marker for IRS (Lawn et al 2005).

\section{Treatment of TB in HIV positive patients}

Current guidelines recommend that irrespective of HIV status, tuberculosis requires a minimum of 6 months of treatment with 4 drugs (including rifampin) in the intensive phase and 2 drugs in the continuation phase. Treatment consists of INH (H), Rifampin (RIF), Ethambutol (EMB) and Pyrazinamide (PYZ) for 2 months followed by INH and RIF for 4 months, given either daily or intermittently. In India, under the RNTCP, patients with newly diagnosed TB receive a 6-month thrice-weekly regimen (Cat I-2EHRZ/
$4 \mathrm{RH}_{3}$ ) while those with relapse, default or failure receive an 8-month regimen (Cat II - $2 \mathrm{SEHRZ}_{3} / 1 \mathrm{EHRZ}_{3} / 5 \mathrm{EHR}_{3}$ ). Patients with smear negative pulmonary TB and localized extrapulmonary forms are treated with Cat III - $2 \mathrm{HRZ}_{3} /$ $4 \mathrm{RH}_{3}$ however this regimen should be avoided in persons with HIV infection.

While there have been no randomized clinical trials comparing daily and thrice-weekly treatment, observational studies have documented a higher risk of acquired rifamycin resistance when highly intermittent regimens are used in patients with advanced HIV disease (Nahid et al 2007). Factors that have been associated with higher failure and relapse rates along with increased mortality during treatment and follow-up are severe immunosuppression (CD4 < 100 cells $/ \mathrm{mm}^{3}$ ), highly intermittent rifampicin administration, lack of rifampicin in the regimen used and duration of treatment of $<6$ months. (Ackah et al 1995; Driver et al 2001; Jindani et al 2004; Lawn et al 2007a, b; Nahid et al 2007). The Tuberculosis Research Centre (TRC) found, in a pilot study of patients with advanced HIV and $\mathrm{TB}$, a favourable response of $72 \%$ and an unfavourable response of $28 \%$ (including treatment failure and deaths) when treated with RNTCP Cat I regimen. Further, $37 \%$ of patients had died within 24 months of completing ATT. In general, HIV-infected patients with TB have higher mortality during and after treatment compared to HIV uninfected patients (Harries et al 2001; Nahid et al 2007; Swaminathan et al 2008). An ongoing randomized clinical trial at TRC is examining the benefits of a longer (9-month) regimen in reducing recurrences. Wider access to anti-retroviral treatment will help reduce mortality due to HIVTB.

\section{Anti-retroviral treatment}

Currently available antiretroviral agents fall in the following groups of drugs: Nucleoside reverse transcriptase inhibitors (NRTIs), Non-nucleoside reverse transcriptase inhibitor (NNRTI's), Protease inhibitors (PI), Integrase inhibitors, Chemokine receptor antagonists and Entry (fusion) inhibitors. HAART refers to the use of a combination of at least 3 antiretroviral (ART) drugs to form a maximally suppressive regimen. Regimens recommended for use in India for patients with concomitant $\mathrm{TB}$ are a combination of 2 NRTI's with Efavirenz or less commonly Nevirapine. The NRTI combinations used commonly are Zidovudine with Lamivudine, Stavudine with Lamivudine, Tenofovir with Lamivudine, and rarely Abacavir with Lamivudine or Didanosine with Lamivudine (only the first two combinations are available in the national program) (National AIDS Control Organization 2007) There is some preliminary data to suggest that nevirapine can be safely given with rifampicin, but results of ongoing clinical trials are awaited 
(Manosuthi et al 2006, 2008; Cohen et al 2008). Among the PI's, nelfinavir, saquinavir, lopinavir and ritonavir can be used but additive hepatoxicity may occur with anti-TB drugs requiring close monitoring of liver function. (de Jong et al 2004).

Table 1 summarizes the WHO recommendations for timing of antiretroviral treatment initiation in HIVTB patients. In our experience, about $2 / 3 \mathrm{rd}$ of HIV infected individuals have a CD4 $<200$ cells $/ \mathrm{mm}^{3}$ when they present with TB. Hence, the earlier use of ART is likely to reduce mortality and morbidity in this group of patients. Table 2 compares the advantages of early versus deferred ART in patients with TB. While the risks of IRIS and overlapping drug toxicities are present, the major advantage of early ART is the significant reduction in deaths.

\section{Rifampicin-ART interactions and cytochrome P450 - 3A (CYP-3A) system}

Rifamycins are unique in their ability to destroy both intracellular and intermittently and slowly growing TB bacilli and therefore form an indispensable component of anti-TB regimens. Non-Rifampicin containing regimens used to treat HIV-TB are associated with greater failure and relapse rates. (Jindani et al 2004; de Jong et al 2004). However, Rifamycins induce the CYP-3A enzyme system in the liver and intestinal wall, thereby increasing the metabolism of PI and non-nucleoside reverse transcriptase inhibitors (table 3 ). The effect is weaker with rifabutin than rifampin. Rifampin is metabolized through deacetylation and is not itself affected by the CYP-3A system. When rifampicin and antiretroviral drugs are given together, decreased trough levels may result, leading to therapeutic failure. Nevirapine levels are reduced by about $40-55 \%$, efavirenz by $25 \%$ and delavaridine by $96 \%$ (McIlleron et al 2007). It has been suggested that the dose of Efavirenz be increased to $800 \mathrm{mg}$ when administered along with Rifampicin, but this may not be necessary in subjects weighing $<50 \mathrm{~kg}$. In India, Efavirenz is the preferred drug for use in HIV-TB co infected individuals at the standard $600 \mathrm{mg}$ dose. However, Nevirapine may be used in HIV-TB patients on concomitant ATT, when Efavirenz is contraindicated as in pregnancy, persons with psychiatric problems or due to toxicity. Alternatively, ATT may be modified with Rifabutin replacing rifampicin with the former given at a dose of $300 \mathrm{mg}$ OD twice/thrice-weekly with nevirapinebased ART.

\section{Toxicity}

The overlapping toxicities due to the drugs commonly used for HIV associated TB are shown in table 4. Peripheral neuropathy which can be due to HIV per se can be further aggravated by Isoniazid, Stavudine and ddI. Almost all the

Table 2. Advantages of early vs deferred ART

\begin{tabular}{ll}
\hline Early ART & Deferred ART \\
\hline $\begin{array}{l}\text { Early mortality due to TB is } \\
\text { reduced. }\end{array}$ & Decrease in toxicity \\
$\begin{array}{l}\text { Improvement in cure rate, } \\
\text { reduction in relapses }\end{array}$ & Drug interaction reduced. \\
$\begin{array}{l}\text { Drug resistance caused } \\
\text { by malabsorption can be } \\
\text { minimized. }\end{array}$ & Decrease in IRS \\
$\begin{array}{l}\text { Prevents other OI's } \\
\text { simultaneously }\end{array}$ & $\begin{array}{l}\text { ATT period can be used to } \\
\text { lay a foundation for better } \\
\text { adherence to ART }\end{array}$ \\
$\begin{array}{l}\text { Period of Hospitalization } \\
\text { reduced }\end{array}$ & $\begin{array}{l}\text { Management of ATT toxicity } \\
\text { is crucial for subsequent ART } \\
\text { adherence }\end{array}$ \\
\hline
\end{tabular}

Table 1. WHO recommendations for starting ART in HIV-TB patients on ATT (World Health Organization 2006)

\begin{tabular}{lll}
\hline CD4 cell count & ART recommendations & $\begin{array}{l}\text { Timing of ART in relation to start of TB } \\
\text { treatment }\end{array}$ \\
CD4 $<200$ cells $/ \mathrm{mm}^{3}$ & Recommend ART & Between two and eight weeks \\
CD4 between 200 and 350 cells $/ \mathrm{mm}^{3}$ & Recommend ART & After eight weeks \\
CD4 $>350$ cells $/ \mathrm{mm}^{3}$ & Defer ART $^{\mathrm{b}}$ & $\begin{array}{l}\text { Re-evaluate patient at eight weeks and at } \\
\text { the end of TB treatment }\end{array}$ \\
Not available & Recommend ART & Between two and eight weeks \\
\hline
\end{tabular}

${ }^{a}$ An EFV-containing regimen is the preferred first-line regimen. Alternative first-line treatment regimens include NVP or triple NRTI (based on TDF or ABC) regimens. For NVP-containing regimens, ALT should be checked at 4, 8 and 12 weeks; treatment should be decided on the basis of symptoms thereafter.

${ }^{\mathrm{b}}$ ART should start as soon as TB treatment is tolerated, particularly in patients with severe immunosuppression.

'ART should be started if other non-TB stage 3 or 4 events are present.

${ }^{\mathrm{d}}$ For some TB diagnoses that generally respond well to anti-TB therapy (ie, lymph node TB, uncomplicated pleural effusion), deferral of ART should be considered. 
Table 3. Drug interactions of antiretroviral drugs with Rifampicin and Rifabutin

\begin{tabular}{|c|c|c|}
\hline & Rifampicin (normal daily dose $600 \mathrm{mg}$ ) & $\begin{array}{l}\text { Rifabutin (daily, unless mentioned; normal } \\
\text { daily dose } 300 \mathrm{mg} \text { ) }\end{array}$ \\
\hline NRTIs & Normal rifampicin dose & Normal rifabutin dose \\
\hline \multicolumn{3}{|l|}{ NNRTIs } \\
\hline Efavirenz & Efavirenz dose at $600 \mathrm{mg}$ & Increase rifabutin dose to $450-600 \mathrm{mg}$ \\
\hline \multicolumn{3}{|l|}{ Normal efavirenz dose } \\
\hline Nevirapine & $\begin{array}{l}\text { Probably can be used at normal nevirapine } \\
\text { dose (more data required) }\end{array}$ & Normal dose each \\
\hline Delavirdine & NR (not recommended) & NR \\
\hline \multicolumn{3}{|l|}{ Protease inhibitors } \\
\hline Amprenavir / Nelfinavir & NR & Lower rifabutin dose to $150 \mathrm{mg}$ \\
\hline Atazanavir & No data & $\begin{array}{l}\text { Lower rifabutin dose to } 150 \mathrm{mg} \text { three times } \\
\text { weekly }\end{array}$ \\
\hline Saquinavir & Can use with low-dose ritinavir "boost" & Normal rifabutin dose \\
\hline Ritonavir & Use only with full-dose ritonavir & $\begin{array}{l}\text { Lower rifabutin dose to } 150 \mathrm{mg} \text { two times } \\
\text { weekly }\end{array}$ \\
\hline Lopinavir / ritonavir & $\begin{array}{l}\text { Probably can be used with lopinavir / } \\
\text { rotonavir dose increase to } 400 / 400 \mathrm{mg} \text { or } \\
800 / 200 \mathrm{mg}\end{array}$ & $\begin{array}{l}\text { Lower rifabutin dose to } 150 \mathrm{mg} \text { three times } \\
\text { weekly }\end{array}$ \\
\hline
\end{tabular}

Modified from de Jong et al (2004).

Table 4. Overlapping toxicities between ATT and ART

\begin{tabular}{lll}
\hline & ATT & ART \\
\hline Hepatoxicity & H, R, Z, & NVP, NRTI's \\
Neuropathy & H & ddI, d4T, \\
Hyperuricemia & Z, E & ddI \\
Neuropsyciatric disturbances & H & EFZ \\
GI symptoms & All & All, especially PI's \\
Skin rashes & $\mathrm{R}, \mathrm{Z}$ & NVP, EFZ, \\
& & nelfinavir, Abacavir \\
Hematological & $\mathrm{R}$ & ZDV, PI's \\
Perioral numbness & $\mathrm{Sm}$ & Amprenavir \\
Nephrotoxic & $\mathrm{R}$, E, Sm & ddI \\
\hline
\end{tabular}

drugs used are potentially hepatotoxic but the combination of Nevirapine and Rifampicin needs to be monitored closely. Thiacetazone should not be used in HIV+ individuals because of the risk of Stevens-Johnson syndrome. Co-trimoxazole can also produce cutaneous toxicity and has to be considered in the differential diagnosis of a skin rash. While Efavirenz can produce giddiness and sleep disturbances in the first few days/weeks of administration, rarely life-threatening psychiatric manifestations (depression, suicidal tendencies) can occur which require immediate termination of the drug. Most adverse effects of drugs are mild and can be managed symptomatically.

\section{Anti-TB drug resistance}

While overall patterns of drug resistance to first line antiTB drugs are similar in HIV positive and negative patients, MDRTB is marginally higher (3-4\%) in HIV positive patients with newly diagnosed TB (Swaminathan 2005). Rifampicin mono- resistance is more common in HIV infected patients and arises independently from mutations in drug susceptible strains. The mechanism is unclear but may be due to malabsorption of anti-TB drugs. Treatment for MDR-TB should ideally be based on drug susceptibility patterns and should employ at least 3-4 new drugs. The regimen should include an aminoglycoside and be given under direct observation. A typical regimen would consist of Inj Streptomycin or Kanamycin (three times a week) along with a combination of Ofloxacin, Ethambutol, Ethionamide, Pyrazinamide with or without Cycloserine or PAS given daily. The treatment should be continued for 18-24 months (for at least 12 to 18 months after the cultures have become negative). Outcome of MDR - TB in HIV is poor. A study from South Africa in 2003 has revealed 41\% mortality among HIV-infected MDR-TB cases. In the New York study in 1996, the situation was no less different with $72 \%$ of MDR -TB cases with HIV dying during treatment. (Finlay et al 2004; Park et al 1996).

Recently, strains of M. tuberculosis labelled as extensively drug resistant (XDR) TB have emerged and spread rapidly through HIV-infected populations leading to high mortality (Andrews et al 2007). XDRTB has been reported from India 
also (Singh et al 2007; Thomas et al 2007), and basically is a result of mismanagement of TB. XDRTB is practically untreatable and the best approach, therefore, is to prevent its emergence, by strengthening and supporting the TB control program.

\section{TB-IRIS}

Immune reconstitution inflammatory syndrome (IRIS) is defined as transient worsening or appearance of new symptoms, signs or radiographic manifestations after initiation of HAART. Tuberculosis is the most frequent pathogen associated with IRIS, of which, lymph node enlargement is the commonest manifestation. In one study, the incidence of IRIS in TB alone was $2 \%$, with HIV coinfection it was $7 \%$, and in those started on HAART it was $36 \%$ (Narita et al 1998). The median period of IRIS presentation is approximtely 4-6 weeks after starting ART, however IRIS has been observed as early as one week after starting ART. Risk factors include high viral load, very low CD4 and initiation of ART soon after TB diagnosis. In TB IRIS, fever is the most frequent symptom, along with worsening infiltrates on chest $\mathrm{x}$-ray. Other manifestations include enlargement and liquefaction of lymph nodes, appearance of fresh crops of nodes, pleural effusion, pericardial effusion, ascites, subcutaneous abscesses, expanding CNS lesions and visceral lesions (Burman and Jones 2001).

Two types of IRIS are observed

(i) An unmasking of a previously latent or sub-clinical infection precipitated by HAART - induced immunologic changes occurring within 1-4 weeks of initiation of HAART (e.g.) MAC lymphadenitis, TB lymphadenitis and cryptococcal meningitis.

(ii) Augmented host response to antigens present in low amounts in tissues (paradoxial response).

Risk factors for TB -IRIS include early initiation of ART within two months of starting ATT, low CD4, presence of extrapulmonary TB especially of the central nervous system, disseminated TB and high viral load (Lawn et al 2007a, b). The pathogenesis of IRIS includes a sharp amplification of Interferon-gamma producing mycobacteria-specific $\mathrm{T}$ cells along with an acute outpouring of Th1 cytokines (Borgarit 2006). It is likely that CD4 $+\mathrm{T}$ cells are not the only mediators of IRIS; therefore an increase in CD4 cell count with HAART does not necessarily correlate with the development of IRIS (Manabe et al 2007). Hypercalcemia is a strange but frequent manifestation in TB-related IRIS. It is probably related to increased production of 125 dihydroxy cholecalceferol in functioning granulomas. A simple way of demonstrating IRIS is restoration of DTH response to PPD. Interferon gamma secretion and cell mediated immune response to mycobacteria are restored leading to restoration of DTH skin test response to mycobacterial antigens. Thus HAART reverses the impact of HIV on the host granulomatous response to mycobacteria. But the rapidly compensating inflammatory response damages the tissues that characterize IRIS. (Lawn et al 2005)

The differential diagnoses include treatment failure, progression of TB, superadded infections and drug reaction. The best way to tackle IRS is to rule out and treat common, endemic causes of fever first. Most IRIS responds to nonsteroidal anti-inflammatory drugs (NSAIDS) along with continuation of ART. A course of steroids (Prednisolone 1 $\mathrm{mg} / \mathrm{kg}$ body weight) tapered over 4-6 weeks may be required when fever/constitutional symptoms prevail despite NSAID treatment or life-threatening symptoms occur. Thalidomide and pentoxyfilline have also been tried as anti-inflammatory agents (Lawn et al 2005). Rarely, ART needs to be terminated.

\section{Preventive therapy (Chemoprophylaxis) for TB in India}

Several randomized clinical trials in HIV-infected persons have shown that the incidence of TB can be reduced by $40-60 \%$ by preventive therapy (Woldehanna and Volmink 2004; Mohammed et al 2007). The standard regimen is Isoniazid for 12 months, though several short-course regimens like $2 \mathrm{RZ}$ and $3 \mathrm{RH}$ have been tried and found to be equivalent. However, adverse reactions tend to be higher with multi-drug regimens and the $2 \mathrm{RZ}$ regimen is no longer recommended because of a high incidence of hepatotoxicity, including several fatal cases in HIV un-infected persons (Narita et al 2002). Standard recommendations in USA, UK etc include giving preventive therapy to all PPD-positive ( $>5$ $\mathrm{mm}$ ) HIV positive persons, after ruling out active TB.

Presently, there is no national policy regarding TB preventive therapy for HIV positive persons in India. A clinical trial being conducted at the Tuberculosis Research Centre, Chennai is investigating two different regimens: a 6month regimen of ethambutol and INH vs. a 3-year regimen of INH alone, in order to establish the ideal duration of therapy. In a TB-endemic country like India, consideration must be given to providing preventive therapy to HIVinfected persons regardless of Mantoux status.. This is because the prevalence of TB in the community is high with a constant risk of exposure as well as the fact that the Mantoux test has many pitfalls in interpretation. TB preventive therapy should form part of the care and support package for HIV+ persons that includes co-trimoxazole prophylaxis, nutritional counseling and supplementation and anti-retroviral treatment. It needs to be delivered through integrated counseling centres or anti-retroviral treatment centres, where HIV+ individuals are followed over the long term. 


\section{Coordination between TB and AIDS control programs}

At the national level, there is a concerted effort to achieve coordination between the designated microscopy centre (DMC) of the RNTCP and the integrated counselling and testing centers (ICTC) of the HIV/AIDS control program. The objective is to promote early diagnosis and treatment of TB in HIV infected individuals and vice versa. All ICTC clients with suggestive symptoms are referred by the counselor to the nearest microscopy centre for investigations to rule out TB. In 2007, approximately 5\% of all diagnosed TB cases in India came from ICTCs, demonstrating that these are excellent sites for active TB case finding (RNTCP report 2008). Further, the yield of cases was similar (approximately $20 \%$ ) from HIV infected and uninfected clients. Counselors need ongoing training to improve their knowledge about TB diagnosis and treatment. One of the programmatic limitations encountered is the lack of investigations for extrapulmonary TB at peripheral health facilities; another is the distance between DMCs and ICTCs in some districts. The system works much better when both are located in the same site.

Till a few months ago, TB patients were offered HIV testing only when the physician suspected HIV, due to coexisting symptoms or signs (selective testing). Recently, the National AIDS Control Organization has taken a decision to routinely offer HIV testing to all diagnosed TB patients in the high-prevalence states. Our study on 4000 TB patients demonstrated that over $2 / 3$ rds were willing to undergo an HIV test and the major barrier to acceptance was patients not perceiving themselves to be at risk (Thomas et al 2007). If patients are counseled and explained the importance of having an HIV test, when they are diagnosed with TB, most will accept the test.

With increasing availability of ART across the country, diagnosis of HIV is beneficial to the individual as he/she can be referred to the nearest ART centre for evaluation and initiation of antiretroviral treatment, if indicated. Tuberculosis clinics, therefore, form an important entry point for HIV diagnosis, care and support. Further, the counseling that patients receive as part of the process of testing will help prevent new infections in the future. Coordination and crosstalk between these two government health programs is crucial not only to improve the outcome of HIV-infected TB patients but also to control the burden of tuberculosis in India.

\section{References}

Ackah A N, Coulibaly D, Digbeu H, Diallo K, Vetter K M, Coulibaly I M, Greenberg A E, De Cock K M 1995 Response to treatment, mortality and CD4 lymphocyte counts in HIV infected persons with tuberculosis in Abidjan, Cote d'Ivoire; Lancet 345 607-610
Agarwal S P and Chauhan L S 2005 Tuberculosis control in India (Elsevier, a division of Reed Elsevier India Private Limited)

Ahmad Z and Shameem M 2005 Manifestations of Tuberculosis in HIV Infected Patients; JIACM 6 302-305

Andrews J R, Shah N S, Gandhi N, Moll T, Friedland G; Tugela Ferry Care and Research (TF CARES) Collaboration 2007 Multidrug-resistant and extensively drug-resistant tuberculosis: implications for the HIV epidemic and antiretroviral therapy rollout in South Africa; J. Infect. Dis. (Suppl. 3) 196 S482-S490

Antonucci G, Girardi E, Raviglione M C, Ippolito G 1995 Risk factors for tuberculosis in HIV-infected persons: A prospective cohort study; JAMA 274 143-148

Arora V K and Kumar S V 1999 Pattern of opportunistic pulmonary infections in HIV sero-positive subjects: observations from Pondicherry, India; Indian J. Chest Dis. Allied Sci. 41 135-144

Burman W J and Jones B E 2001 Treatment of HIV-related Tuberculosis in the Era of Effective Antiretroviral Therapy; Am. J. Respir. Crit. Care Med. 164 7-12

Cohen K, van Cutsem G, Boulle A, McIlleron H, Goemaere E, Smith P J and Maartens G 2008 Effect of rifampicin-based antitubercular therapy on nevirapine plasma concentrations in South African adults with HIV-associated tuberculosis; $J$. Antimicrob. Chemother. 61 389-393

De Cock K M, Soro B, Coulibaly I M and Lucas S B 1992 Tuberculosis and HIV infection in sub-Saharan Africa; JAMA 268 1581-1587

de Jong B C, Israelski D M, Corbett E L and Small P M 2004 Clinical management of tuberculosis in the context of HIV infection; Annu. Rev. Med. 55 283-301

Deivanayagam C N, Rajasekaran S, Senthilnathan V, Krishnarajasekhar R, Raja K, Chandrasekar C, Palanisamy S, Samuel Dinesh A, Jothivel G and Elango S V 2001 Clinicoradiological spectrum of tuberculosis among HIV sero-positives - a Tambaram study; Indian J. Tuber. 48 123-127

Driver C R, Munsiff S S, Li J, Kundamal N and Osahan S S 2001 Relapse in persons treated for drug susceptible tuberculosis in a population with high co-infection with Human Immunodeficiency virus in New York City; Clin. Infect. Dis. 33 $1762-1769$

Emergence of XDR-TB WHO concern over extensive drug resistant TB strains that are virtually untreatable 2006; accessed from http://www.who.int/mediacentre/news/notes/2006/np23/ en/print.html on 25/05/08

Fee M J, Oo M M, Gabayan A E, Radin D R and Barnes P F 1995 Abdominal tuberculosis in patients infected with the human immunodeficiency virus; Clin. Infect. Dis. 20 938-944

Finlay A F, Walt M V, Holtz T H, Thorpe L E, Wells C D and Weyer K 2004 Treatment outcome of patients with multidrug resistant tuberculosis in south Africa using a standardized regimen, 19992000 (abstract 584); in Program and abstracts of the infectious diseases (Society of America 2004 Annual Meeting Alexandria , VA: infectious Disease society of America) p. 155

Gil-Setas A, Torroba L, Fernandez J L, Martinez-Artola V and Olite J 2004 Evaluation of the MB/BacT system compared with Middlebrook 7H11 and Lowenstein-Jensen media for detection and recovery of mycobacteria from clinical specimens; Clin. Microbiol. Infect. 10 224-228 
Harries A D, Hargreaves N J, Jindani A, Enarson D A, Meher D and Salaniponi F M 2001 Deaths from tuberculosis in Sub-Saharan African countries with a high prevalence of HIV -1; Lancet 357 $1519-1523$

Havlir D V and Barnes P F 1999 Tuberculosis in patients with human immunodeficiency virus infection; N. Engl. J. Med. 340 $367-373$

Jindani A, Nunn A J and Enarson D A 2004 Two 8-month regimens of chemotherapy for treatment of newly diagnosed pulmonary tuberculosis: international multicentre randomized trial; Lancet 364 1244-1251

Kejariwal D, Sarkar N, Chakraborti S K, Agarwal V and Roy S 2001 Pyrexia of unknown origin: a prospective study of 100 cases; J. Postgrad. Med. 47 104-107

Kumarasamy N, Vallabhaneni S, Flanigan T P, Mayer K H and Solomon S 2005 Clinical profile of HIV in India; Indian J. Med. Res. 121 377-394

Lawn S D, Bekker L G and Miller R F 2005 Immune reconstitution disease associated with mycobacterial infections in HIVinfected individuals receiving antiretrovirals; Lancet Infect. Dis. 5 361-373

Lawn S D, Butera S B and Shinnick T M 2002 Tuberculosis unleashed: the impact of HIV infection on the host granulomatous response to mycobacterium tuberculosis; Microbes Infection 4 635-646

Lawn S D, Myer L, Bekker L G and Wood R 2007 Tuberculosisassociated immune reconstitution disease: incidence, risk factors and impact in an antiretroviral treatment service in South Africa; AIDS 21 335-341

Lawn S, Myer L, Bekker L G and Wood R 2007 Early mortality among patients with HIV associated TB in Africa: Implications for the time to initiate $A R T$ (Conference on Retroviruses and Opportunistic Infections: Abstract 81)

Lee J J, Suo J, Lin C B, Wang J D, Lin T Y and Tsai Y C 2003 Comparative evaluation of the BACTEC MGIT 960 system with solid medium for isolation of mycobacteria; Int. J. Tuber. Lung Dis. 7 569-574

Long R, Maycher B, Scalcini M and Manfred J 1991 Chest roentgenogram in pulmonary tuberculosis patients seropositive for HIV type I; Chest 99 123-127

Lucas S B, De Cock K M, Hounnou A, Peacock C, Diomande M, Hondé M, Beaumel A, Kestens L and Kadio A 1994 Contribution of tuberculosis to slim disease in Africa; BMJ 308 1531-1533

Manabe Y C, Campbell J D, Sydnor E and Moore R D 2007 Immune reconstitution inflammatory syndrome: risk factors and treatment implications; J. Acquir. Immune Defici. Syndr. $46456-462$

Manosuthi W, Mankatitham W, Lueangniyomkul A, Chimsuntorn S and Sungkanuparph S 2008 Standard-dose efavirenz vs. standard-dose nevirapine in antiretroviral regimens among HIV1 and tuberculosis co-infected patients who received rifampicin; HIV Med. 9 294-299

Manosuthi W, Sungkanuparph S, Thakkinstian A, Rattanasiri S, Chaovavanich A, Prasithsirikul W, Likanonsakul S and Ruxrungtham K 2006 Plasma nevirapine levels and 24-week efficacy in HIV-infected patients receiving nevirapine-based highly active antiretroviral therapy with or without rifampicin; Clin. Infect. Dis. 43 253-255
McIlleron H, Meintjes G, Burman W J and Maartens G 2007 Complications of antiretroviral therapy in patients with tuberculosis: drug interactions, toxicity, and immune reconstitution inflammatory syndrome; J. Infect. Dis. (Suppl. 1) 196 S63-S75

Miranda A, Morgan M, Jamal L, Laserson K, Barreira D, Silva G, Santos J, Wells C, Paine P and Garrett D 2007 Impact of antiretroviral therapy on the incidence of tuber-culosis: the Brazilian experience, 1995-2001; PLOS ONE 2 e826

Mohammed A, Myre L, Ehrlich R, Wood R, Cilliers F and Maartens G 2007 Randomised controlled trial of isoniazid preventive therapy in South African adults with advanced HIV disease; Int. J. Tuber. Lung Dis. 11 1114-1120

Nahid P, Gonzalez L C, Rudoy I, De long B C, Unger A and Kawamura L M 2007 Treatment outcomes of patients with HIV and tuberculosis; Am. J. Respire. Crit. Care Med. 175 1196-1206

Narita M, Ashkin D, Hollender E S and Pitchenik A E 1998 Paradoxical worsening of tuberculosis following Antiretroviral therapy in patients with AIDS; Am. Crit. Care 158 157-161

Narita M, Kellman M, Franchini D L, McMillan M E, Hollender E S and Ashkin D 2002 Short course rifampicin and pyrazinamide treatment for latent tuberculosis infection in patients with HIV infection: the 2 year experience of a comprehensive community based programme in Broward county, Florida; Chest 122 $1292-1298$

National AIDS Control Organization 2007 Antiretroviral therapy guidelines for HIV-infected adults and adolescents including post-exposure prophylaxis (Section A7)

Park M M, Davis A L, Schluger N W, Cohen H and Rom W N 1996 Outcome of MDR -TB patients, 1983-1993: prolonged survival with appropriate therapy; Am. J. Respir. Crit. Care Med. 153 317-324

Ramachandran R, Swaminathan S, Somasundaram S, Asgar V N, Paramesh P and Paramasivan C N 2002 Mycobacteremia in tuberculosis patients with HIV infection; Indian J. Tuberc. 50 29-31

Rieder H L 1999 Epidemiological basis of tuberculosis control First edition [International Union against Tuberculosis and Lung Diseases (UNION)] pp 50-52

Rupali P, Abraham O C, Zachariah A, Subramanian S and Mathai D 2003 Aetiology of prolonged fever in antiretroviral-naïve human immunodeficiency virus-infected adults; Natl. Med. J. India 16 193-199

Sahn S A and Neff T A 1974 Miliary tuberculosis; Am. J. Med. 56 494-505

Sharma S K, Mohan A and Kadhiravan T 2005 HIV-TB coinfection: epidemiology, diagnosis \& management; Indian J. Med. Res. 121 550-567

Singh S, Gopinath K, Shahdad S, Kaur M, Singh B and Sharma P 2007 Nontuberculous mycobacterial infections in Indian AIDS patients detected by a novel set of ESAT- 6 polymerase chain reaction primers; Jpn. J. Infect. Dis. 60 14-18

Singh S, Sankar M M and Gopinath K 2007 High rate of extensively drug-resistant tuberculosis in Indian AIDS patients; AIDS 21 2345-2347

Steingart K R, Henry M, Ng V, Hopewell P C, Ramsay A, Cunningham J, Urbanczik R, Perkins M, Aziz M A and Pai M 2006 Fluorescence versus conventional sputum smear 
microscopy for tuberculosis: a systematic review; Lancet Infect. Dis. 6 570-581

Swaminathan S, Deivanayagam C N, Rajasekaran S, Venkatesan P, Padmapriyadarsini C, Menon P A, Ponnuraja C and Dilip M 2008 Long term follow up of HIV-infected patients with tuberculosis treated with 6-month intermittent short course chemotherapy; Natl. Med. J. Indian 21 3-7

Swaminathan S, Narendran G, Menon P A, Padmapriyadarsini C, Arunkumar N, Sudharshanam N M, Ramesh Kumar S and Chandrasekhar S 2007 Impact of HIV infection on radiographic features in patients with pulmonary tuberculosis; Indian J. Chest Dis. Allied Sci. 49 133-136

Swaminathan S, Paramasivan C N, Ponnuraja C, Iliayas S, Rajasekaran S and Narayanan P R 2005 Anti-tuberculosis drug resistance tuberculosis in South India; Int. J. Tuberc. Lung Dis. 9 896-900

Swaminathan S, Ramachandran R, Baskaran G, Paramasivan C N, Ramanathan U, Venkatesan P, Prabhakar R and Datta M 2000 Risk of development of tuberculosis in HIV infected patients; Int. J. Tuberc. Lung Dis. 4 839-844

Swaminathan S, Subbaraman R, Venkatesan P, Subramanyam S, Ramesh Kumar S, Mayer K H and Narayanan P R 2008 Tuberculin skin test results in HIV-infected patients in India: Implications for latent tuberculosis treatment; Int. J. Tuberc. Lung Dis. 12 168-173

TB/HIV, A Clinical Manual, WHO 2004 accessed from http://libdoc.who.int/publications/2004/9241546344.pdf on 25/ 05/2008
Thomas A, Ramachandran R, Rehaman F, Jaggarajamma K, Santha T, Selvakumar N, Krishnan N, Mohan N S, Sundaram V, Wares F and Narayanan PR 2007 Management of multi drug resistance tuberculosis in the field: Tuberculosis Research Centre experience; Indian J. Tuberc. 54 117-124

Thomas B E, Ramachandran R, Anitha S, Swaminathan S 2007 Feasibility of routine HIV testing among TB patients through a Voluntary, Counseling and Testing Centre (VCTC); Int. J. Tuberc. Lung Dis. 11 1296-1301

WHO Smear Negative Algorithm 2006 Improving the diagnosis and treatment of smear-negative pulmonary and extrapulmonary tuberculosis among adults and adolescents; Accessed from http:/ /www.who.int/tb/publications/2006/tbhiv_recommendations.pdf

William R and Stuart G 2004 Tuberculosis (New York: Lippin Cott Williams and Wilkins)

Williams-Bouyer N, Yorke R, Lee H I and Woods G L 2000 Comparison of the BACTEC MGIT 960 and ESP culture system II for growth and detection of mycobacteria; J. Clin. Microbiol. 38 4167-4170

Woldehanna S and Volmink J 2004 Treatment of latent tuberculosis infection in HIV infected persons; Cochrane Database Syst. Rev. 1 CD000171

World Health Organization 2006 Initiating First-Line ART in Relationship to Starting Anti-TB Therapy; accessed from http: $/ / w w w$. womenchildrenhiv.org/wchiv? page $=$ charts-00-13 on $25 / 05 / 2008$

World Health Organization 2008. Global tuberculosis control: surveillance, planning, financing. WHO report 2008. WHO/ HTM/TB/2008.393. Geneva

ePublication: 15 October 2008 